\title{
Les tombeaux de saints musulmans au Xinjiang
}

Culte, réforme, histoire

\section{Alexandre Papas}

\section{CpenEdition}

Journals

Édition électronique

URL : http://journals.openedition.org/assr/13633

DOI : $10.4000 /$ assr. 13633

ISSN : $1777-5825$

Éditeur

Éditions de l'EHESS

\section{Édition imprimée}

Date de publication : 1 juin 2008

Pagination : 47-62

ISBN : 978-2-7132-2190-3

ISSN : 0335-5985

\section{Référence électronique}

Alexandre Papas, "Les tombeaux de saints musulmans au Xinjiang », Archives de sciences sociales des religions [En ligne], 142 | avril-juin 2008, mis en ligne le 20 octobre 2011, consulté le 30 avril 2019. URL : http://journals.openedition.org/assr/13633 ; DOI : 10.4000/assr.13633 


\section{Alexandre Papas}

\section{Les tombeaux de saints musulmans au Xinjiang Culte, réforme, histoire}

Depuis le début des années 1980, le paysage funéraire sacré du Xinjiang ${ }^{1}$ connaît un changement paradoxal : de grands mausolées abritant les dépouilles de saints musulmans sont érigés ou bien restaurés à grands frais, arborant des coupoles neuves et des céramiques étincelantes qui font oublier l'état de ruines ou de déliquescence dans lequel certains de ces lieux se trouvaient encore il y a quelques décennies. Des tombes surgissent ou ressurgissent ici et là, plus ou moins majestueuses, plus ou moins modestes. Masami Hamada note ainsi qu'un grand nombre de tombes saintes ont été découvertes et exhumées durant ces années (Hamada, 2004 : 1034-1038). Aujourd'hui, ces mêmes lieux, certains d'entre eux du moins, sont en passe de devenir ou redevenir des foyers de vie religieuse voire des lieux publics comme tels. S'agirait-il d'un revirement de la politique de Pékin envers la minorité ouigoure et ses traditions sociales et religieuses ? S'agit-il d'un acte de contrition après les destructions de la Révolution culturelle qui n'épargnèrent pas l'islam des frontières du Nord-Ouest ? Ou bien d'une forme de revivalisme minoritaire et spirituel consenti par un État chinois réputé assimilationniste et athée ? Malgré les apparences séductrices, au moins pour les fidèles, de ce paysage nouveau, l'histoire et l'anthropologie du culte des saints musulmans au Xinjiang ou Turkestan oriental rappellent à quel point la prudence d'analyse est de rigueur et quelles sont les continuités qui donnent un sens aux phénomènes récents ${ }^{2}$.

Je commencerai par quelques rappels de l'histoire et du rôle historique du culte des saints et de la sainteté en islam centre-asiatique, en particulier au Turkestan oriental, afin de mieux comprendre le contexte religieux dans lequel s'impliquent

1. La Région autonome oügoure du Xinjiang $\left(1646000 \mathrm{~km}^{2}\right)$, située dans la partie occidentale de la République populaire de Chine, est peuplée en majorité d'Ouigours (env. $8000000)$, population turcophone et seconde minorité musulmane en Chine après celle des Hui (musulmans de langue chinoise).

2. Un constat et un questionnement comparables à ceux-ci valent pour la Région autonome du Tibet (Kapstein, 2004). 
les autorités chinoises et leurs réformes sécularistes. Cette implication est historiquement marquée, suivant une période et des changements ou des variations voire des paradoxes - comme dans le cas de l'alliance objective des communistes chinois et des réformistes musulmans. Je tâcherai d'expliquer cet aspect depuis 1949, c'est-à-dire à partir de l'instauration de la République populaire jusqu'à aujourd'hui. Enfin, je terminerai par une double hypothèse : la première concerne la pérennité de la sainteté et de son culte au Xinjiang malgré un demi-siècle de réformes hostiles et de signes avant-coureurs inquiétants. La seconde voudrait tirer du cas centre-asiatique, ici étudié dans la durée, un enseignement d'anthropologie historique tâchant de penser la tombe sainte comme lieu de mémoire historique, au-delà de l'opposition entre culte et réformes.

\section{Les saints musulmans et leur culte au Xinjiang}

Suivant la profession de foi elle-même (arabe shahâda) ${ }^{3}$ stipulant qu' "il n'y a de Dieu sinon Dieu et Muhammad est son Prophète ", l'islam est un monothéisme rigoureux, peut-être le plus rigoureux de tous ; si Muhammad est - toujours selon la formule - le Prophète accompli de Dieu, le sceau même de la Prophétie (ar. khâtam al-nubuwwa) ${ }^{4}$, interdisant la possibilité de tout prochain agent de la Révélation, il n'en demeure pas moins que l'islam comprend des intermédiaires entre Allah et ses fidèles, intermédiaires du nom de walî (pluriel ar. awliyâ : amis, proches) c'est-à-dire les saints (Chodkiewicz, 1995). Or ceux-là sont innombrables puisqu'à la différence du christianisme catholique, par exemple ${ }^{5}$, aucun procès de canonisation n'existe, la sainteté étant laissée à l'expression de la vox populi. C'est dire si le culte des saints représente une véritable institution dans les sociétés musulmanes et c'est dire aussi à quel point il est répandu à travers tout le monde musulman. Outre cette dimension décisive de l'opinion populaire dans la sainteté, une autre particularité explique le succès et l'importance de son culte : elle tient à la licence religieuse, c'est-à-dire à certaines libertés ou émancipations que le fidèle se permet à l'occasion du culte des saints et tout spécialement sur les lieux de ce culte, les tombes de saints - mazâr dans les différentes langues d'Asie centrale. Forte présence des femmes ; pratiques propitiatoires, prophylactiques, syncrétiques; ou encore activités festives (musiques, danses, jeux et même foire ${ }^{6}$ ) sont caractéristiques des mausolées. Les lieux saints

\footnotetext{
3. Abréviations : ar. pour arabe ; ou. pour ouigour ; ch. pour chinois (transl. pinyin).

4. Ou Sceau des Prophètes (khâtam al-nabiyyîn) selon Coran, XXXIII, 40. Pour une analyse théologique de cette notion, voir Van Ess, 1991 : 29-30. La mystique médiévale glose également cette notion (Radkte \& O'Kane, 1996 : 106-107).

5. Il faudrait nuancer cette assertion tant il est vrai que " dans toute communauté religieuse se crée une tension entre ce que l'autorité, d'une part, et ce que différents groupes ou individus, d'autre part, définissent comme sainteté sans consulter l'autorité (...) »(Kleinberg, $2005: 16$ ).

6. Un exemple contemporain intéressant de ces activités festives est celui du grand pèlerinage au mazâr Imâm 'Âsim non loin de la ville de Lop à l'est de Khotan. Un autre exemple
} 
(ar. mashâhid) représentent, plus que les grands sanctuaires panislamiques (Ka‘ba à La Mecque, mosquée du Prophète à Médine et Haram al-Sharîf à Jérusalem) et davantage que les mosquées, des lieux d'expression de la ferveur religieuse ${ }^{7}$.

Bref, le culte des saints en islam apparaît comme une institution plurielle, complexe mais omniprésente, à l'exception - mais qui confirme la règle - du royaume wahhabite saoudien. Au contraire de ce dernier, et comme toutes les régions d'islam finalement, l'Asie centrale, notamment chinoise, a accueilli puis cultivé la sainteté de façon continue dans l'histoire de son islamisation (Castagné, 1951 ; DeWeese, 2005 : 126-129).

Selon une vision traditionnelle des historiens, l'islamisation de la région prend son essor officiel sous la dynastie turk des Karakhanides avec la conversion à l'islam de Satûq Bughrâ Khân en $934^{8}$. Il ne faut pas déduire trop rapidement de cet épisode un simple processus d'islamisation par le haut. Il faut plutôt retenir l'idée d'un phénomène double: un travail de conversion de l'élite dirigeante d'une part, et de l'autre, des campagnes de conversions de masse à travers les oasis. Néanmoins, cette conversion de Satûq Bughrâ Khân, qui a même donné lieu à un hadith du Prophète rigoureusement apocryphe disant que "Satûq est [ou sera] le premier Turk à devenir musulman ", mène l'islam au statut de religion d'État, au nom de laquelle le pouvoir politique favorise, précisément, l'édification de tombeaux de saints. À la mort de Satûq en 955, son fils et successeur érige un tombeau que les fidèles peuvent encore visiter aujourd'hui, sis à Artush (à une quarantaine de kilomètres au nord de Kashgar $)^{9}$. Ce lieu fera l'objet d'un culte tout au long de l'histoire du Turkestan oriental (Dawut, 2001 : 1-6), en particulier aux $\mathrm{XVI}^{\mathrm{e}}, \mathrm{XVII}^{\mathrm{e}}$ et $\mathrm{XVIII}{ }^{\mathrm{e}}$ siècles qui correspondent à la seconde période clé dans la formation du culte des saints.

La période des Khwâja (XVI ${ }^{\mathrm{e}}-\mathrm{XVIII}{ }^{\mathrm{e}}$ siècles) permet une nouvelle impulsion dans la mesure où ces Khwâja sont des prêcheurs soufis, appartenant à l'ordre (ar. tarîqa) de la Naqshbandiyya, qui défend et développe - à l'instar d'autres ordres soufis - une mystique fondée sur la sainteté. Le saint représente ici l'idéal

serait celui du mazâr Ordam Pâdishâh, situé entre Kashgar et Yengisar, décrit dans Harris \& Dawut, 2002 et Sawada, 2002. Mais ce second pèlerinage a été interdit en 1997. On retrouve ce phénomène festif ailleurs dans le monde musulman, un exemple particulièrement bien étudié étant celui du mawlid (pèlerinage pour l'anniversaire de la naissance) de Sayyid al-Badawî à Tanta en Égypte (Mayeur-Jaouen, 2004).

7. En suivant les distinctions proposées dans Grabar, 2005. Voir aussi Mayeur-Jaouen, 2000 .

8. Il existe une riche littérature sur la figure de Satûq Bughrâ Khân : Grenard, 1900 ; Bartol'd, 1968 : 257-261 ; Baldick, 1993 ; DeWeese, 1996 ; Hamada, 2002. Rappelons que cet événement et l'existence même de Satûq Bughrâ font l'objet de controverses parmi les spécialistes. Dans une perspective plus générale, les récits de conversion à l'islam dans la tradition centre-asiatique ont été étudiés dans DeWeese, 1994. L'islamisation du bassin du Tarim est un sujet complexe qui ne se résume évidemment pas à cette conversion. Celle-ci représente néanmoins une étape importante dans l'histoire du culte des saints musulmans.

9. Le mausolée actuel ne date que de 1954 mais il a été construit sur les ruines du précédent. 
du cheminement mystique et le modèle même de la religiosité sunnite. C'est dire, à nouveau, l'importance du saint et de son culte. Ainsi de l'arrivée d'Ishâq Khwâja en 1580 jusqu'au décès d'Âfâq Khwâja en 1694, une institutionnalisation du culte des saints se met en place autour de leur tombeau. Des mausolées sont construits ou restaurés, le rite se fixe à peu près avec circumambulation (ar. tawâf; ou. tavap) ${ }^{10}$, ex-voto, pèlerinage et dévotions ; puis ces lieux deviennent de véritables pôles de la vie tant religieuse que sociale et politique (Papas, 2005 : 51-54, 140-145). Pour reprendre l'exemple du tombeau de Satûq Bughrâ Khân, ce lieu saint, tout en attirant différentes pratiques dévotionnelles devient un lieu de rassemblement populaire et d'activisme politique puisqu'à partir de lui sont initiés des mouvements de jihâd ou des cérémonies d'allégeance royale (Papas, $2005: 32,43,67,228)$. La tombe sainte acquiert donc une charge symbolique extrêmement importante.

Or c'est bien cette charge que souhaite exploiter Ya'qûb Beg, fondateur de l'émirat du Turkestan oriental entre 1864 et 1877 (Kim, 2004). Cette troisième période se caractérise par une promotion politico-religieuse des lieux saints à travers les sept villes (ou. yettishahr) qui composent l'émirat : Kashgar, Yengisar, Yarkand, Khotan, Aqsu, Kucha et Korla. Biens de mainmorte (ar. waqf) et donations pieuses obligatoires permettent de réaménager de nombreuses mosquées, comme la fameuse grande mosquée (ou. jamä mäsjid) Idkah de Kashgar (Tursun, 2001 : 54) ; ils financent aussi l'entretien des mausolées importants comme ceux des Sept Muhammad à Yarkand, d'Ordam Pâdishâh à l'est de Yengisar, d'Âfâq Khwâja et, à nouveau, de Satûq Bughrâ Khân; Ya'qûb Beg y pratique en personne des pèlerinages ostentatoires à l'aide de dévotions fastueuses (Zarcone, 1999). Ce faisant, le souverain cherche à capter le charisme des saints et à s'afficher comme l'héritier et le continuateur de leur mandat politico-religieux. Les mazâr, en cette fin du XIX ${ }^{\mathrm{e}}$ siècle, font figure d'institutions du pouvoir.

En faisant à nouveau un saut dans le temps, jusqu'à l'époque actuelle et plus particulièrement durant ces vingt dernières années, le paysage funéraire sacré du Xinjiang connaît une certaine évolution qui prend toutes les apparences d'un renouveau contemporain de la sainteté. Et cela à plusieurs niveaux de manifestation. D'abord, sur le plan de la connaissance : à travers des organes de l'Académie des sciences comme, par exemple l'Institut de Recherche des Religions mondiales, des études, des publications (articles et ouvrages, scientifiques ou même romanesques) se sont multipliées ces derniers temps au Xinjiang, écrites en chinois mais surtout en ouigour, qui traitent des saints et des lieux saints. Ceci est un fait récent dans la production intellectuelle de la région. Ensuite, en second niveau, de grands travaux de restauration sont menés sur plusieurs tombeaux alors même

10. Calqué sur la circumambulation autour de la Ka'ba, le tawâfltavap représente une pratique centrale dans le culte des saints, au point de nommer les mausolées, dans les sources historiques centre-asiatiques en persan, tawâfgâh (litt. lieu de circumambulation). Aujourd'hui encore, le tavap est une pratique dévotionnelle extrêmement répandue au Xinjiang (Häbibullah, $2000: 346-348)$. 
que ceux-là demeuraient parfois à l'état de ruines depuis le début du $\mathrm{XX}^{\mathrm{c}}$ siècle (Stein, 2007 : ill. 18, 52, 53, 301, etc.). Enfin, il s'organise une véritable promotion culturelle de ces tombeaux et des figures historiques qui y reposent, une promotion sans précédent de la part de la République populaire qui utilise les médias et divers moyens publicitaires. Le tombeau de Satûq Bughrâ Khân rassemble de façon exemplaire ces différents degrés et représente aujourd'hui un haut lieu culturel du Xinjiang ${ }^{11}$.

La question qui surgit dès lors est celle de savoir si un haut lieu culturel peut être dans le même temps un haut lieu religieux ; autrement dit, la reconstruction des saints tombeaux ne correspondrait-elle pas plutôt à leur destruction symbolique ? S'agit-il d'une désacralisation masquée ? Pour y répondre, il est nécessaire de remonter à l'histoire du rapport qu'entretient l'État communiste chinois avec le culte des saints musulmans dans la province du Xinjiang, en examinant les politiques imposées par le premier à l'encontre du second.

\section{Contre le culte des mazâr depuis 1949}

Si les autorités chinoises se sont inquiétées du pouvoir des tombeaux dès avant 1949, il reste que l'instauration de la République populaire inaugura une politique antireligieuse qui visait, en particulier, les lieux saints. Oscillant entre athéisme et sécularisme socialiste, changeant d'attitude suivant les époques, les autorités de Pékin ont toutefois été moins féroces qu'on ne pourrait le croire visà-vis des musulmans du Xinjiang. D'autre part, elles ne furent pas les seules à agir contre le culte des saints. Elles furent même appuyées, idéologiquement sinon politiquement, par une intelligentsia ouigoure pétrie des idées du réformisme musulman centre-asiatique ou djadidisme (Dudoignon, 1996 ; Khalid, 1998). Enfin, récente caractéristique de la politique chinoise envers le champ funéraire sacré, l'enjeu d'un patrimoine culturel national change sensiblement la donne. La politique ouigouro-musulmane de l'État chinois ${ }^{12}$, marquée par des

11. Pour information, on notera deux publications importantes (parmi d'autres) : un roman historique et documenté par l'auteur officiel Säypidin Äzizi (1987) ainsi qu'une traduction ouigoure de l'hagiographie majeure de Satûq (en turki) par Molla Haji (1988). À noter l'importance des tirages pour ces deux ouvrages, ainsi que le fait que Säypidin Äzizi (1915-) est un ancien leader révolutionnaire, commissaire politique et dirigeant du comité central du PCC au Xinjiang. Enfin, sur le plan promotionnel, on constate que la totalité des guides touristiques - toutes langues confondues - concernant la région mentionnent le mausolée comme une attraction majeure, dans une stricte conformité avec les options touristiques indiquées par les agences régionales chinoises. Voir, entre autres, China: A Guidebook to Xinjiang, Urumchi, Xinjiang Educational Press, 1988 ; Judy Bonavia, Route de la Soie. De Xi’an à Kashgar, Genève, Éditions Olizane, 1993 (trad. fr.) ; Silk Road. Central Asia Countries, Tokyo, Ryokojin, 1999 (en japonais).

12. La politique sino-musulmane - c'est-à-dire vis-à-vis des Hui - connut une évolution générale comparable : tolérance postrévolutionnaire, destructions de la Révolution culturelle, reconstructions à partir des années 1980. Dru Gladney a analysé la question des tombeaux de saints chez les Hui (1987). 
52 - ArChives De SCIENCES SOCIALES DES RELIGIONS

revirements et des paradoxes, sera menée par le truchement d'un organe principal, l'Association islamique de Chine, créée en 1953, encadrée par des Hui (musulmans sinophones) et des turcophones (musulmans autochtones du Xinjiang).

Avant de détailler ces différents points, il convient de rappeler le contexte idéologique dans lequel ils interviennent, à savoir la politique des nationalités (ch. minzu) dans la République populaire, et plus particulièrement le cas ouigour. Comme en Union Soviétique, les minorités apparurent comme un problème en droit comme en fait (Dreyer, 1968 ; Bergère, 1979) : il s'agissait d'en respecter la spécificité culturelle et historique tout en prônant un seul et unique mouvement vers l'avènement du socialisme; chaque peuple avait droit à l'autonomie tout en fondant l'État unitaire chinois, tel que le formulaient le Programme Commun de 1949 puis la Constitution de 1954. La contradiction de droit entraîna les balancements et les hésitations des faits : à la mansuétude de la période révolutionnaire ${ }^{13}$, succédèrent l'uniformisation forcée du Grand Bond en Avant, le désastre de la Révolution culturelle, enfin l'assouplissement sous Deng Xiaoping. Pour ce qui concerne les Ouigours et la région autonome éponyme, retenons - en suivant Denise Helly - que la révolution au Xinjiang s'établit de manière progressive, au sens où le Parti communiste chinois retarda la transformation socialiste des rapports sociaux (systèmes agraires et matrimoniaux, coutumes et usages, croyances religieuses) jusqu'en 1955, année de la fondation de la Région autonome et début de la collectivisation généralisée (Helly, 1983). McKenzie montre, lui, que durant les quatre séquences suivantes - Campagne des Cent Fleurs (1956-1957), Campagne anti-droitière (1956-1958), Grand Bond en Avant (1958-1960), modération pragmatique sous Wang Enmao, Premier secrétaire du Parti au Xinjiang (1961-1965) - la politique ouigoure du PCC expérimenta différents traitements de la "minorité », dans un climat de rivalité avec l'Union soviétique frontalière (McKenzie, 1986) ${ }^{14}$. La critique du chauvinisme han se doubla de la "rectification " du nationalisme ouigour ou kazakh, à laquelle succéda l'assimilation au modèle socialiste national chinois. Elle-même fut corrigée par la reconnaissance officielle des minorités stipulant que «les différentes nationalités garderont leurs caractéristiques nationales » ${ }^{15}$. Reconnaissance qui s'interrompit en 1966 et fit place au revirement assimilationniste de la «Grande révolution culturelle prolétarienne ». Les minorités symbolisent alors le nationalisme honni ; le respect des caractéristiques nationales est considéré comme une

13. Pour un témoignage sur cette période et les espoirs qu'elle représenta, voir Davidson, 1959.

14. Notons que cette période fait l'objet d'une chronologie détaillée à travers les Shinjiang tarikhi materialliri [Matériaux d'histoire du Xinjiang], Urumchi, Shinjiang Khälq Näshriyati, 30 ssq. (en fin de chaque volume).

15. Formule prononcée lors de la Conférence sur les nationalités à Pékin entre le 21 avril et le 29 mai 1962, en cohérence avec la déclaration postérieure, le 28 septembre 1962 au $10^{\mathrm{e}}$ Plénum du $8^{\mathrm{e}}$ Comité central du PCC, selon laquelle la voie vers le communisme requerrait une période plus longue pour qu'il fût réalisé. 
ligne réactionnaire bourgeoise au sein du Parti (incarnée notamment par Wang Enmao). Le chaos qui en résulte cesse en 1969, mais ce n'est qu'à la mort de Mao en septembre 76, et lors de la purge des éléments radicaux dans le Comité révolutionnaire du Xinjiang (établi en août 1968) à la fin de l'année 1976, qu'il est à nouveau question de droits minoritaires pour les Ouigours ${ }^{16}$. Dernière date charnière à retenir, 1982 et le $12^{\mathrm{e}}$ Congrès du PCC, sous Deng Xiaoping, marquent une politique d'ouverture vis-à-vis des nationalités. Il apparaît que la politique musulmane de Pékin suit à peu près la même chronologie, la même trajectoire, sinueuse et accidentée, que celles de sa politique relative au minzu ouigour.

Il y a là un parallélisme ${ }^{17}$, si ce n'est un lien direct, dans la mesure où les milieux religieux du Xinjiang, en tant que grands propriétaires terriens grâce aux biens de mainmorte, furent la cible des réformes économiques et sociales qui touchèrent l'ensemble des propriétés religieuses en Chine, notamment bouddhistes. C'est ainsi que l'article 3 de la loi agraire de 1950 stipule que : "Les terres appartenant à des sanctuaires d'ancêtres, temples, monastères, églises, écoles et autres institutions publiques devront être confisquées, mais les terres possédées par les mosquées pourront être conservées par celles-ci selon les circonstances et avec le consentement des musulmans résidant dans les régions d'établissement de ces temples " (Helly, 1983 : 21). Des récits témoignent qu'à partir de 1949, les grands mausolées, victimes d'une série de réformes cherchant à annuler leur sacralité, se voient ôter leur pouvoir attractif. Sont retirés les marques et les symboles dévotionnels tels que les mâts et les bannières (ou. tugh vä äläm), les cornes d'animaux, les bois (ou. shidde), les panses de moutons (ou. tulum) et les divers objets votifs. Sont également limitées les fréquentations des lieux et les occasions de rassemblement. Sont interdits les séjours dans les mazâr tels qu'ils étaient pratiqués soit par des mystiques soufis, soit par des chamanes musulmans (ou. bakhshi) ou encore par des groupes d'ascètes itinérants (ou. qäländär, ashiq). Enfin, les autorités font fermer certains lieux de culte ou même de sociabilité religieuse attenants aux mausolées comme les mosquées, les madrasas (écoles religieuses), les khaniqa ( «couvent ", lieu de réunion soufi) et les tünäkkhana (hostellerie pour pèlerins) où le fidèle pouvait séjourner durant plusieurs jours. Néanmoins, les pèlerinages sont tolérés, certains mausolées sont épargnés, et les desservants du culte ne semblent pas inquiétés. D'autre part, le gouvernement met en place, en 1952, plusieurs mesures protégeant les coutumes religieuses : droit d'organiser les célébrations musulmanes (ou. bayram et mäwlut näbi), abolition des taxes sur le sacrifice d'animaux, participation au financement des mosquées, permission de congé durant les fêtes religieuses. Mais, progressivement, le contrôle des autorités religieuses s'organise, la limitation des institutions

16. Sur toute cette période, voir McMillen, 1979 : 180-303.

17. Un autre parallélisme tient dans la politique de la langue ouigoure (Dwyer, 2005). 
islamiques se resserre, des purges sont pratiquées au sein de la très officielle et docile Association islamique de Chine.

Cette relative tolérance cesse définitivement au moment de la Révolution culturelle (ou. mädäniyät inqilabi) qui fait rage entre 1966 et 1971, malgré la déclaration officielle de fin de la révolution en avril 1969 lors du $9^{\mathrm{e}}$ Congrès du PCC, et même dès le 25 février 1967 dans le cas du Xinjiang. En croisant quelques témoignages recueillis sur le terrain avec les données d'ouvrages ouigours qui décrivent les mazâr du Xinjiang (Dawut, 2001 ; Turan, 2001) ${ }^{18}$, l'historien obtient, en dépit de l'absence de chiffres, un tableau dramatique de cette période. Un slogan terrible et bien connu de l'époque, issu d'un discours prononcé par Lin Biao, le 18 août 1966, déclare qu'il faut éliminer les "Quatre vieilleries " (ch. si jiu; ou. töt kona) : les vieilles idées, la vieille culture, les vieilles coutumes et les vieilles habitudes. En effet, sous les coups des gardes rouges ou des «équipes de propagande " chargées de «Lutter-Critiquer-Réformer » (ch. dou-pi-gai.) - slogan lancé en août 1966 lors du $11^{\mathrm{e}}$ Plénum du $8^{\mathrm{e}}$ Comité central du PCC - la plupart des mausolées subissent des destructions partielles ou totales, l'accès à des lieux de pèlerinages est interdit; enfin, plus grave, les témoins évoquent des exécutions sommaires et des massacres parmi les milieux religieux liés aux mazâr, par exemple à Kashgar ou à Qaraqash dans la région de Khotan ${ }^{19}$. Néanmoins, si le traumatisme qu'il a provoqué est encore sensible aujourd'hui, si la mémoire collective a retenu les récits de martyres (Millward \& Tursun, 2004 : 98 ${ }^{20}$, le moment de la Révolution culturelle peut être considéré comme exceptionnel, tout à fait contraire, finalement, à la politique séculariste de l'État chinois qui reprend dès les années 1970 en réaffirmant la liberté religieuse inscrite dans les constitutions.

Plus cohérent apparaît dès lors ce dernier aspect de la politique religieuse de Pékin qui peut être qualifiée de patrimoniale. Son esprit général s'exprime à travers une notion, ou plus exactement un statut administratif et juridique, celui de «site [relevant] du patrimoine culturel » (ou. mädäniyät yadikarliq orun) ${ }^{21}$. Ce statut, entre lieu de mémoire et monument classé, ne trouve pas de définition juridique précise en ouigour ; quoi qu'il en soit, son application semble assez malléable. Le mazâr relève désormais d'un patrimoine national qui lui-même fait partie d'une construction de la nation ou plutôt de la nationalité, le minzu chinois ou millet en ouigour, conduite par la République populaire depuis le recensement

18. J'ignore s'il existe par ailleurs une "littérature de cicatrice » ouigoure comme il en existe en chinois. Voir cependant Friederich, 1997.

19. Entretiens menés en juillet 2004.

20. "(...) it would be impossible to fully understand the disaffection and separatism among Uyghurs and other Xinjiang nationalities without considering the legacy of the Cultural Revolution".

21. Sur la question du patrimoine en Chine populaire, voir Fresnay, 2001 et 2003, ainsi que Zhang, 2003. Je remercie Françoise Aubin de m’avoir indiqué ces références. 
de $1953^{22}$. Le lieu saint passe d'un traitement antireligieux à un traitement patrimonial qui l'intègre, non plus tant à une croyance à éradiquer, qu'à une culture à conserver voire à muséifier. Il s'agit moins d'une sécularisation, moins d'une désacralisation que d'un projet de sacralisation par l'histoire ou la culture, une sacralisation non plus religieuse mais nationale. Ce serait là un premier paradoxe de la politique de l'État chinois vis-à-vis du funéraire sacré : le statut de "site relevant du patrimoine culturel " révèle le caractère nationaliste de la politique des minorités menée par Pékin, si soucieuse pourtant d'éliminer tout séparatisme, mais qui préfère la sacralité d'une nation et de sa culture à la sacralité d'une foi. Les morts saints, symboles de piété, sanctifiés pour et par leur piété, deviennent symboles de civilisation, sanctifiés pour et par l'État.

Un second paradoxe qui caractérise l'esprit de la politique chinoise envers les pratiques funéraires sacrées est l'alliance objective entre son sécularisme socialiste et le réformisme musulman. En effet, il ne faut pas imaginer que l'opposition au culte des saints soit le seul fait d'un État impie, certes puissant, à l'encontre d'une société uniformément dévote. À observer le demi-siècle qui débute en 1949, communistes chinois et réformistes ouigours partagent un esprit commun. Cette collusion contre-nature concerne autant les musulmans chinois que ouigours : certains travaux récents (Zarcone, 2002 ; Chérif-Chebbi, 2004) montrent, chez les Hui comme chez les Ouïgours, une connivence de vue, parfois même d'action, avec les autorités contre le culte des saints. Le réformisme musulman, classiquement, prône depuis le $\mathrm{XIX}^{\mathrm{e}}$ siècle, un islam purifié de ce qu'il considère comme des déviations ou des superstitions (ar. khurafât, ou. khurapatliq) qui confinent à l'hérésie, toutes choses qu'il voit concentrées dans le culte des tombeaux de saints. Il s'agit, en bref, d'un mouvement de réaction orthodoxe mâtiné de modernisme. Le réformisme musulman ouigour a trouvé ses principaux idéologues en Abduqadir Damulla (1881-1923) et Mämitili Äpändi (1901-1937) ${ }^{23}$, plus récemment, chez un certain Nizamüddin Hüsäyn, décédé il y a quelques années. Chez les uns comme chez l'autre, l'attaque contre le culte des saints est d'une violence qui n'a rien à envier aux discours de l'athéisme militant.

Patrimonialisation et orthodoxie sont les deux réalités paradoxales du sécularisme socialiste et du réformisme musulman qui définissent la politique chinoise quant aux pratiques cultuelles du Xinjiang. Cependant, aujourd'hui, dans les années 2000, à l'issue même de cette politique, ces pratiques témoignent d'une vie religieuse toujours intense, signes non pas d'une résistance populaire mais d'une endurance spirituelle. Au-delà du demi-siècle ici considéré, en examinant

22. Le recensement ne sert pas seulement à comptabiliser la population mais à en distinguer les catégories, et dans le cas chinois comme russo-soviétique, à représenter les ethnies en particulier. Un livre emblématique de cette division ethnique du Xinjiang en millet est celui de Baytur \& Sidiq, 1991.

23. Le réformisme musulman du Xinjiang est un sujet en soi. J'espère publier prochainement une étude sur ces deux penseurs djadids importants et néanmoins mal connus. 
cette question du culte des saints sur une plus longue durée, l'historien retrouve peut-être une problématique classique et non point une caractéristique de la seule modernité. À nouveau donc, il faut se méfier du temps court et du passé récent.

\section{La postmodernité d'un culte}

La situation actuelle des mausolées du Xinjiang ne correspond plus aux années de l'athéisme socialiste; elle ne se trouve pas non plus sur les terres du réformisme salafiste ou wahhabite. D'autre part, elle maintient une continuité dans ses pratiques, dans ses croyances et dans ses représentations. Telle serait la postmodernité du culte des saints au Xinjiang : une politique religieuse ambiguë qui devient gestion du sacré, et la continuation de ce que la modernité avait cru pouvoir abolir - la sainteté elle-même.

La récente promotion des mazâr au rang de "sites relevant du patrimoine culturel ", soit d'articles du patrimoine national, revient à une admission tacite du lieu saint en tant que tel. Comme si les réformistes d'Urumchi autant que les officiels de Pékin ne croyaient plus à une disparition des saints et de leur culte. $\mathrm{Au}$ lieu donc de la sécularisation ou désacralisation, le régime communiste tente de changer le contenu du sacré, d'en modifier l'objet. Dans ce phénomène de permutation, le Xinjiang contemporain retrouve un principe classique de l'histoire du culte des saints, ce que Peter Brown, spécialiste du christianisme antique tardif, appelle des "systèmes rivaux de patronage " (1984: 48). Il s'agissait, pour les évêques du Bas-Empire, de promouvoir certaines tombes de saints pour en tirer une liturgie communautaire et une légitimité qui faisaient d'eux, les évêques, les patrons visibles de la communauté, sous le patronage invisible des saints. Or, au fond, il se joue la même pièce dans la Chine communiste actuelle : la gestion des mazâr par l'État et non plus par une famille sainte ou une autorité religieuse, correspond à une récupération du patronage et à une sorte de recyclage du sacré. Ni tolérance religieuse ni athéisme donc mais une ambiguité profonde qui désacralise en re-sacralisant, qui, finalement, cherche à détourner à son compte un sacré qu'elle renonce à vouloir détruire. Le problème revient à savoir si une telle instrumentalisation est possible, si, par conséquent, les clients des saints - c'est-à-dire les fidèles musulmans ouigours - se laissent si aisément réorientés dans leur piété multiséculaire.

Probablement pas. Somme toute, le religieux a gagné et les saints continuent de trôner dans la pérennité, portés par les foules de pèlerins. En effet, le culte des saints n'a jamais cessé d'exister tout au long de l'histoire de l'islam turkestanais. Et même aux pires heures de l'opposition à la dévotion, les fidèles ont maintenu le culte, ils l'ont adapté aux situations successives et ils ont continué de prêter allégeance à leurs patrons célestes. Il en va de même aujourd'hui. Je terminerai sur ce point en proposant, rapidement, deux modèles contemporains 
de culte des saints tombeaux au Xinjiang qui manifestent une adaptation habile aux contraintes de l'époque ${ }^{24}$.

Le premier - que j'appelle culte discret - consiste à mener des pratiques dévotionnelles non ostentatoires sur les tombeaux. Par exemple, sur un mazâr aussi important que celui d’Âfâq Khwâja dans la banlieue de Kashgar, devenu monument historique, les fidèles continuent de placer des objets et des marques de culte au milieu des touristes qui viennent nombreux et en groupe - pendant certaines périodes de l'année et de façon souvent précipitée ${ }^{25}$. Ainsi, sur l'aile gauche du mausolée se trouve un encensoir, sur l'aile droite la claustra est pour partie obturée par de petits ex-voto faits d'étoffes colorées, à défaut de grandes bannières; d'autre part, les fidèles mènent encore sur ce mazâr des pèlerinages personnels ou familiaux, à défaut d'être communautaires ${ }^{26}$. Bref, discrètement, par endroits et à certains moments soigneusement choisis, la communauté musulmane maintient le culte en dépit de la marchandisation touristique et continue à percevoir le lieu saint, non comme un musée, mais comme une source de baraka, c'est-à-dire de bénédiction. Signe indiscutable de cette résilience, le cimetière qui jouxte le mazâr, immense et surpeuplé, représente le lieu favori d'inhumation pour les familles du district, formant ainsi une véritable nécropole : il demeure important de se faire enterrer non loin de la source de baraka.

Le second modèle de culte est davantage un culte éloigné, ou distant, qui organise l'entretien et la visite de mazâr situés dans des endroits isolés, difficiles d'accès. Je mentionnerai deux exemples pour une même région, celle de Khotan, dans le Sud du Xinjiang. L'un se trouve dans la petite localité de Bäshtoghraq, il s'agit du mazâr de Siyavush, sur lequel pratiquement aucune donnée historique n'existe. La particularité de ce mausolée tient à son environnement naturel puisqu'il est perdu au milieu des frondaisons et envahi par les hautes herbes. Son entrée est à peine visible et le pèlerin doit se frayer un chemin pour y parvenir. Quant au second, il est situé dans le district de la ville de Qaraqash à l'ouest de Khotan, mais en plein désert, à plusieurs kilomètres de toute habitation, sans piste pour y conduire. Le mazâr de Qum Rabat Padishahim correspondrait à un champ de bataille sous les Karakhanides au début du XI siècle, et plus précisément à un épisode martyr de l'islam turkestanais (Stein, 1907 : 119; Grenard,

24. Je me permets de signaler que je prépare, en collaboration avec Lisa Ross, un ouvrage consacré aux lieux saints du Xinjiang. Les brèves descriptions qui suivent seront largement plus développées dans notre livre.

25. L'afflux touristique, comme dans d'autres provinces de Chine, apparaît comme un obstacle évident au culte. La nouveauté la plus préoccupante est, de toute évidence, le prix de l'entrée du mausolée. Toutefois, il faut rappeler que d'autres pèlerinages supposent un certain déboursement - comme c'est le cas du hajj, le pèlerinage à La Mecque. En outre, il faut remarquer un fait : le tourisme empêche définitivement la fermeture du lieu saint devenu attraction touristique, et autorise un tourisme religieux pratiqués par les fidèles, notamment de la part des Hui. Cette identification du pèlerinage au voyage touristique n'est d'ailleurs pas une nouveauté et correspond à une pratique religieuse traditionnelle en islam.

26. Observations personnelles et entretiens en août 1999, juin 2001 et août 2004 . 
1898 : 39). Là, le pèlerin doit, non plus se frayer un chemin, mais tout simplement le trouver ${ }^{27}$. Il faut voir ici le paradoxe que représente cet éloignement de la destination pérégrine dans la mesure où le tombeau demeure lieu public, occasion de familiarité, intimement liée à la vie sociale elle-même. À moins d'y voir cette dialectique de la proximité et de la distance, de la tentative spirituelle et de l'épreuve physique, telle qu'elle fut formulée par Alphonse Dupront (1987: 373-378). Reste que ces deux cas de culte éloigné semblent davantage répondre à des contingences politiques. Fait significatif enfin, ces deux mazâr ne sont pas classés comme "sites relevant du patrimoine culturel».

\section{Conclusion : la tombe et le temps}

Il semble que le paysage funéraire sacré du Xinjiang ne se laisse pas si aisément réformer et que, face aux variations ou aux hésitations de l'État, les croyants mènent, dans le rite même, moins une résistance politique qu'une constance religieuse. Il ne s'agit pas, bien sûr, de retomber dans les grandes Histoires des religions qui présumaient une "éternelle sacralité du rapport des hommes aux lieux de manifestation, de célébration ou d'invocation du divin (...) »; il ne s'agit pas non plus de recourir aux "grandes catégories d'usage courant en sociologie des religions sur les "pôles" constitutifs du religieux " (Iogna-Prat \& Veinstein, 2005 : 388). En revanche, une analyse anthropologique peut s'aventurer à comprendre cette économie rituelle sur les tombes, y compris les tentatives de réformes qu'elle suscite, comme une certaine expérience du temps historique convoquée par les morts. Loyauté envers les saints patrons d'un côté, re-sacralisation civile de l'autre, ces morts particuliers appellent une intervention matérielle (tellurique, architecturale, scripturale) qui veut interpréter le temps et donner un sens à l'histoire. Remontant sans cesse, à rythmes différents, dans le passé - que ce soit pour la formation du culte des saints musulmans dès le $\mathrm{X}^{\mathrm{e}}$ siècle, pour sa répression à partir de 1949 , ou encore à propos du principe de rivalité des patronages sur le temps long - les saints inhumés ${ }^{28}$ imposaient de formuler, selon les périodes et les acteurs, une signification historique précise : amorce de l'islamisation, faîte des lignées mystiques, marquage de l'État, contradiction de la modernité ou du progrès, fin de l'histoire, conservation du patrimoine, mutation du culte ${ }^{29}$. Les morts aussi, illustres du moins, écrivent l'histoire des sociétés.

27. Observations personnelles et entretiens en juillet 2004.

28. Présents ou absents du reste, puisqu'il est souvent difficile de distinguer entre tombes et cénotaphes.

29. Dans un autre contexte, ottoman médiéval, Gilles Veinstein observe que les conquêtes d'une nouvelle province s'accompagnèrent fréquemment de la promotion d'un saint tombeau sis en cette province : par le recours aux tombes sacrées, il s'agissait d'identifier comme islamiques et l'origine mythique et le destin irréversible du territoire conquis (Veinstein, 2005). 
Les tombes de saints ne se réduisent pas à une sédimentation de croyances archaïques survivant aux réformes successives du monothéisme et du sécularisme, elles ne sont pas non plus de simples lieux de mémoire stricto sensu. Elles apparaissent davantage comme des objets irréductibles et signifiants pour ces sociétés qui inhument leurs grands hommes, au sens où les tombeaux signalent des temps d'histoire et figurent leurs différentes occurrences. Si Philippe Ariès nota bien, dans son ouvrage classique L'homme devant la mort (1977 : 207-211), le caractère exceptionnel des tombes de saints (laïques autant que religieux) - expliquant comment elles "échappèrent » à l'anonymat funéraire commun et comment elles activèrent l'apparition des épitaphes pour l'ensemble des corps enfouis, mouvement qui inaugura la mémoire obituaire - il manqua peut-être une dimension plus anthropologique qui ressort clairement du cas musulman centre-asiatique. Car il ne s'agit pas seulement, en milieu sépulcral, de répondre "à un souci de faire mémoire " selon l'expression d'Ariès. Il est davantage question de dire l'histoire. Tout se passe en effet comme si la tombe, érigée, saccagée, détruite, rétablie, maquillée, à chaque occasion investie par une demande de sens du temps, en formulait le récit matériel.

Alexandre PAPAS

Centre National de la Recherche Scientifique, Paris alex.p@club-internet.fr

\section{Bibliographie}

ARIÈs Philippe, 1977, L’homme devant la mort. I. Le temps des gisants, Paris, Seuil. ÄzIZI Säypidin, 1987, Sutuq Bughrakhan, Pékin, Millätlär Näshriyati.

BALDICK Julian, 1993, Imaginary Muslims. The Uwaysi Sufis of Central Asia, New York, New York University Press.

Bartol'D Vassilij V., 1968, Turkestan. Down to the Mongol Invasion, Londres, Luzac $\&$ Co ( $3^{\mathrm{c}}$ édition).

BAYTUR Änvär, SIDIQ Khäyzinsa, 1991, Shinjiangdiki millätlärning tarikhi [Histoire des nationalités du Xinjiang], Pékin, Millätlär Näshriyati.

BERGÈrE Marie-Claire, 1979, "L'influence du modèle soviétique sur la politique des minorités nationales en Chine : le cas du Sinkiang (1949-1962) ", Revue française de science politique, 3, pp. 402-425.

BRown Peter, 1984, Le culte des saints. Son essor et sa fonction dans la chrétienté latine, Paris, Éditions du Cerf.

Castagné Joseph, 1951, "Le culte des lieux saints de l'Islam au Turkestan ", L'Ethnographie, 46, pp. 46-124.

Chérif-Chebbi Leila, 2004, "Brothers and Comrades: Muslim Fundamentalists and Communists Allied for the Transmission of Islamic Knowledge in China ", in Dudoignon S. (éd.), Devout Societies vs. Impious States? Transmitting Islamic Learning in Russia, Central Asia and China, through the Twentieth Century, Berlin, Klaus Schwarz Verlag, coll. "Islamkundliche Untersuchungen ", 258, pp. 61-90.

ChODKIEwicz Michel, 1995, "La sainteté et les saints en islam » in Chambert-Loir H., Guillot C., (éds.), Le culte des saints dans le monde musulman, Paris, École Française d'Extrême-Orient, coll. "Études thématiques " 4, pp. 13-32. 
60 - ARCHIVES DE SCIENCES SOCIALES DES RELIGIONS

DAvidson Basil, 1959, Turkestan vivant, Paris, René Julliard.

DAwUT Rahilä, 2001, Uyghur mazarliri [Les mausolées ouigours], Urumchi, Shinjang Khälq Näshriyati.

DeWeEse Devin, 1994, Islamization and Native Religion in the Golden Horde. Baba Tükles and Conversion to Islam in Historical and Epic Tradition, University Park, The Pennsylvania State University Press.

DEWesse Devin, 1996, "The Tadhkira-i Bughrā-khān and the "Uvaysī” Sufis of Central Asia: Notes in Review of Imaginary Muslims ", Central Asiatic Journal, 40/1, pp. 87-127.

-, 2005, "Walī, 5. Central Asia », Encyclopédie de l’islam, XI, $2^{\mathrm{e}}$ éd., Leyde, Brill, pp. 126-129.

Dreyer June, 1968, "China's Minority Nationalities in the Cultural Revolution ", The China Quarterly, 35, pp. 96-109.

Dudoignon Stéphane (éd.), 1996, "Le Réformisme musulman en Asie centrale. Du "premier renouveau" à la soviétisation 1788-1937 ", Cahiers du Monde russe, XXXVII/1-2.

Dupront Alphonse, 1987, Du Sacré. Croisades et pèlerinages. Images et langages, Paris, Gallimard.

DWYer Arienne M., 2005, The Xinjiang Conflict: Uyghur Identity, Language Policy, and Political Discourse, Washington, East-West Center Washington, coll. "Policy Studies» 15.

FRESNAIS Jocelyne, 2001, La protection du patrimoine en République populaire de Chine, 1949-1999, Paris, CNRS Éditions.

-, 2001, "Cinquante ans de gestion du patrimoine architectural en République populaire de Chine : 1949-1999 ", in Gravari-Barbas M., Guichard-Anguis S., (éds.), Regards croisés sur le patrimoine dans le monde à l'aube $d u$ XXI ${ }^{e}$ siècle, Paris, Presses de l’Université de Paris-Sorbonne, pp. 77-97.

FRIEDERICH Michael, 1997, Die ujghurische Literatur in Xinjiang 1956-1966, Wiesbaden, Otto Harrassowitz.

GLADNEY Dru, 1987, « Muslim Tombs and Ethnic Folklore: Charters for Hui Identity », The Journal of Asian Studies, 46-3, pp. 495-532.

Grabar Oleg, 2005, "Le mosquée et le sanctuaire. Sainteté des lieux en Islam ", Revue de l'histoire des religions, 222/4, pp. 481-489.

GrenARD Fernand, 1898, "Le Turkestan et le Tibet: Étude ethnographique et sociologique " in Dutreuil de Rhins J.-L., Mission scientifique dans la Haute Asie 18901895, vol. 2, Paris, Ernest Leroux.

GRENARD Fernand, 1900, «La légende de Satok Boghra Khân », Journal Asiatique, IX-15, pp. 5-79.

HÄBıBulla Abdurähim, 2001, Uyghur Etnografisi [Ethnographie ouigoure], Urumchi, Shinjiang Khälq Näshriyati.

HAjı Mola, 1988, Bughrakhanlar täzkirisi [Mémorial des Bughrakhanides], Qäshqär, Qäshqär Uyghur Näshriyati.

HAMAda Masami, 2002, "Le mausolée et le culte de Satuq Bughrâ Khân ", Journal d'histoire du soufisme, 3, pp. 62-87.

-, 2004 "Le pouvoir des lieux saints dans le Turkestan oriental », Annales. Histoire, Sciences Sociales, 5-6, pp. 1019-1040.

Harris Rachel, DAwUT Rahilä, 2002, "Mazar Festivals of the Uyghurs: Music, Islam and the Chinese State ", British Journal of Ethnomusicology, 11-1, pp. 101-118.

Helly Denise, 1983, "Le parti communiste chinois et la question ethnique : le cas des Uygurs du Xinjiang 1949-1960 ", Études chinoises. Bulletin de l'Association française d'études chinoise, 2, pp. 3-35. 
Iogna-Prat Dominique, Veinstein Gilles, 2005, "Lieux de culte, lieux saints dans le judaisme, le christianisme et l'islam. Présentation ", Revue de l'histoire des religions, 222-4, pp. 387-391.

KapsTeIN Matthew T., 2004, "A Thorn in the Dragon's Side. Tibetan Buddhist culture in China ", in Rossabi M., (éd.), Governing China's Multiethnic Frontiers, SeattleLondres, University of Washington Press, pp. 230-269.

Khalid Adeeb, 1998, The Politics of Muslim Cultural Reform: Jadidism in Central Asia, Berkeley-Los Angeles, University of California Press.

KIM Hodong, 2004, Holy War in China: The Muslim Rebellion and State in Chinese Central Asia, 1864-1877, Stanford, Stanford University Press.

Kleinberg Aviad, 2005, Histoire de saints. Leur rôle dans la formation de l'Occident, Paris, Gallimard.

Mayeur-Jaouen Catherine, 2000, "Tombeau, mosquée et zâwiya : la polarité des lieux saints musulmans ", in Vauchez A. (éd.), Lieux sacrés, lieux de culte, sanctuaires, Rome, École française de Rome, pp. 133-147.

-, 2004, Histoire d'un pèlerinage légendaire en islam. Le mouled de Tantâ du XIII siècle à nos jours, Paris, Aubier.

McKenzie Éric F., 1986, Chinese Communist Policy toward Minority Nationalities in the Xinjiang-Uighur Autonomous Region in the Context of the Sino-Soviet Dispute, 1956-1965, Ph.D., Charlottesville, University of Virginia, 521 p., inédite.

McMillen Donald H., 1979, Chinese Communist Power and Policy in Xinjiang, 19491977, Boulder, West View Press.

Millward James, Tursun Nabijan, 2004, «Political History and Strategies of Control, 1884-1978 ", in Starr S.F., (éd.), Xinjiang: China’s Muslim Borderland, New York, M.E. Sharpe, pp. 63-98.

Papas Alexandre, 2005, Soufisme et politique entre Chine, Tibet et Turkestan. Étude sur les Khwajas Naqshbandis du Turkestan oriental, Paris, Jean Maisonneuve.

RADTKE Bernd, O'KANE John, 1996, The Concept of Sainthood in Early Islamic Mysticism. Two Works by Al-Hakim Al-Tirmidhî, Richmond, Curzon Press.

SAWAda Minoru, 2002, "A Study of the Current Ordam-Padishah System ", Journal d'histoire du soufisme, 3, pp. 89-111.

STEIN Aurel, 1907, Ancient Khotan. Detailed Report of Archeological Explorations in Chinese Turkestan, 1-2, Oxford, Clarendon Press.

TURAN Adil Muhämmät, 2001, Qäshqärdiki qädimi izlar [Les vestiges anciens de Kashgar], Urumchi, Shinjiang Khälq Näshriyati.

TURSUN Hüsäynjan, 2001, "Qäshqär heyitgah jamäsi [La mosquée Idkah de Kashgar] », Junggo musulmanliri [Musulmans de Chine], 1, p. 54.

VAN Ess Josef, 1991, Theologie und Gesellschaft im 2. und 3. Jahrhundert Hidschra. Eine Geschichte des religiösen Denkens im frühen Islam. Band I, Berlin-New York, Walter de Gruyter.

Veinstein Gilles, 2005, "Le rôle des tombes sacrées dans la conquête ottomane ", Revue de l'histoire des religions, 222-4, pp. 509-528.

ZARCONE Thierry, 1999, "Quand le saint légitime le politique: le mausolée d'Afaq Khwâja à Kashgar », Central Asian Survey, 18, pp. 225-241.

ZARCONE Thierry, 2002, "Le culte des saints au Xinjiang de 1949 à nos jours ", Journal d'histoire du soufisme, 3, pp. 133-172.

ZHANG Liang, 2003, La naissance $d u$ concept de patrimoine en Chine, XIX ${ }^{e}-X^{e}$ siècles, Paris, Éditions Recherches/Ipraus. 
62 - ARCHIVES DE SCIENCES SOCIALES DES RELIGIONS

\section{Résumé}

Cet article analyse la situation actuelle $d u$ culte des saints chez les musulmans oügours de Chine. Les mausolées du Xinjiang font aujourd'hui l'objet d'une attention bienveillante de la part des autorités chinoises alors même qu'ils étaient considérés comme un contre-pouvoir à l'idéologie révolutionnaire. À la lumière de l'anthropologie historique et religieuse, cette apparente contradiction trouve sa cohérence. Le culte des saints, malgré les tentatives successives de réformes, d'inspirations non seulement socialistes mais islamiques se maintient au prix d'une métamorphose des rituels. L'État a donc décidé d'intégrer les lieux saints au patrimoine national, un geste non dénué d'ambiguïté. Mais au-delà de ce statut officiel, les figures saintes et leurs tombeaux se voient à nouveau confiés un rôle social et symbolique à titre de lieux de mémoire historique.

Mots-clés : Saints, islam, Xinjiang, tombeaux, réforme.

\section{Abstract}

This article examines the present situation of the cult of saints among the Uyghur Muslims in China. Today, Chinese authorities give favourable consideration to the mausoleums in Xinjiang, whereas these places were perceived as counter-powers to the revolutionary ideology. In the light of historical and religious anthropology, this apparent contradiction finds its consistency. Despite the successive attempts of reforms, whether socialist or islamic, the cult of saints remains after ritual metamorphosis. The State has therefore decided to integrate the holy places into the national patrimony, a rather ambivalent gesture. Yet, beyond this official status, the saintly figures and their tombs are assigned again a social and symbolic role, in so far as they became places of historical memory.

Key words: Saints, Islam, Xinjiang, graves, reform.

\section{Resumen}

Este artículo analiza la situación actual del culto a los santos entre los musulmanes oigures de China. Los mausoleos de Xingiiang son hoy en día objeto de la atención protectora de las autoridades chinas, siendo antes considerados como un contrapoder a la ideología revolucionaria. A la luz de la antropología histórica y religiosa, esta aparente contradicción se vuelve coherente. El culto de los santos, aún con los sucesivos intentos de reformas, de inspiración no sólo socialista sino islámica, se mantiene al precio de una metamorfosis de los rituales. El estado ha entonces decidido integrar los lugares santos al patrimonio nacional, un gesto no exento de ambigüedad. Pero más allá de este estatuto oficial, las figuras santas y sus tumbas son nuevamente depositarias de un rol social y simbólivo a título de lugar de memoria histórica.

Palabras clave: Santos, Islam, Xinjiang, tombas, reforma. 